\title{
A SOLAR MODEL WITH TURBULENT DIFFUSION MIXING : SURFACE ABUNDANCES AND OSCILLATIONS
}

\author{
Y. Lebreton (1), G. Berthomieu (2), J. Provost ${ }^{(2)}$ \\ (1) Geneva Observatory \\ CH-1290 Sauverny \\ (2) Nice Observatory and CNRS (UA128) \\ B.P. 139 \\ F-06003 Nice Cedex
}

\begin{abstract}
A solar model which includes the plausible presence of a mild mixing in the solar inner radiative zone is presented. The mixing is supposed to result from the hydrodynamical instabilities which may occur in a differentially rotating medium leading then to turbulent diffusion. The model includes up-to-date input physics and takes into account the inhibition of the turbulent motions, due to molecular weight gradients (i.e. for $\left.M_{r} / M_{\odot}<0.5\right)$. The properties of the model are compared to the standard model properties and to observational results: this turbulent diffusion mixing (TDM) improves the results on surface abundances of the light elements $\left({ }^{7} \mathrm{Li},{ }^{3} \mathrm{He}\right)$ and does not reduce the solar neutrino flux relative to the standard model. Moreover preliminary results show that this TDM weakly modifies the oscillation frequencies.
\end{abstract}

\section{SOLAR MODELS DESCRIPTION}

The properties of two solar models, concerning both the oscillations and the surface abundances of ${ }^{8}$ He and ${ }^{7} \mathrm{Li}$, are discussed and compared to the observations. Both models were computed with the Geneva stellar evolutionary code developped by A. Maeder and co-workers. Up-to-date input physics is used and TDM is included in one of the models. The other model, a standard one, has been caleulated for purpose of comparison.

TDM in the radiative stellar layers has been originally proposed by Schatzman (1977) in order to solve the problem of the observed stellar surface abundances of the light elements $\left({ }^{7} \mathrm{Li},{ }^{9} \mathrm{Be},{ }^{8} \mathrm{He}\right)$, moreover it also seemed to be a possible process in the search of a solution for the solar neutrino problem. It is treated here according to Zahn's scheme, in particular the inhibition of the diffusion by a mean molecnlar weight gradient is taken into account.

The thermal imbalance, settling in in rotating stars, generates meridional circulation currents, which maintain differential rotation in the star. This velocity field is shear unstable on equipotential surfaces, which leads to a two-dimensional turbulence at large scales. From the usual cascade of turbulence towards small scales may result a three-dimensional tail of turbulence (this has been shown in a laboratory experiment by Hoptinger et al., 1982). This 3-D tail of the 2-D turbulence is responsible, according to Zahn (1983) of the vertical diffusion of chemical elements in the stars. The turbulent diffusion coefficient is then drawn from Sweet's solution for meridional circulations and the turbulence is inhibited in a given region of the star as soon as the molecular weight gradient exceeds some critical value. 
In the region where diffusion takes place, the turbulent diffusion coefficient is taken to be proportional to the molecular viscosity $\nu$, that is $D=R e^{*} \nu$. The following approximation of $R e^{*}: R e^{*}=8 r / H_{p}$ (see Lebreton and Maeder, 1986b) is used. It is a rather crude approximation but it is in good agreement with the various estimations of $R e^{*}$ derived from different solar constraints by Berthomieu et al. (1983), as shown in the Figure 1.

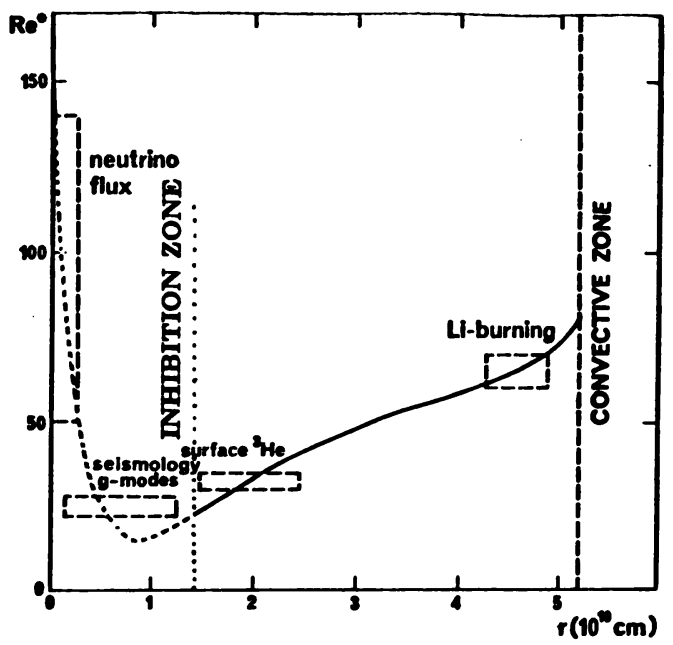

Figure 1: Variation of $R e^{*}$ as function of the radius. The zone where the diffusion is inhibited at solar age is indicated as well as the main observational constraints.

Changes of chemical composition in the sun are calculated for each chemical species which enters either in p-p chain or CNO cycle. Both nuclear reactions and transport by turbulent diffusion are taken into account. The nuclear reaction rates are from Harris et al. (1983) and Fowler et al. (1975). In the standard solar model we nsed Däppen's treatment (1980) for the equation of state. We used Los Alamos opacity tables of Huebner et al. (1977) completed by molecular absorption coefificients at low temperatures (i.e. for $k T<1 e V$ ). The models have an initial helinm abundance $Y=0.28$ in mass fraction and an initial relative metal abondance $Z=0.019$. The mixing length ratio used in the treatment of convection is $l / H_{p}=1.9$. The increase of $\mathrm{Y}$ and of $l / H_{p}$ relative to previous values (see e.g. Schatzman and Maeder, 1981) is mainly due to a change in the used opacity data (Lebreton and Maeder, 1986a).

\section{MAIN RESULTS AT SOLAR AGE $\left(t_{\odot} \sim 4.610^{9} \mathrm{yr}\right)$}

The main properties of the two models are summarized in Table 1.

$\begin{array}{ccccc}\text { model } & L / L_{\odot} & R / R_{\odot} & N_{\nu S N U} & D_{\text {conv }} \\ \text { utandard } & 1.000 & 1.018 & 13 & 1.7610^{10} \\ \text { aiffused } & 0.098 & 1.015 & 13 & 1.8010^{10}\end{array}$

Table 1: Luminosity, radius,neutrino flux, depth of the onter convection zone.

The solar nentrino $\mathrm{fux}$ is the same in both models, that is more than 6 times greater than the observed one. This high value is mainly due to the high initial helium content of 
the models. Inhibition of the diffusion by the $\mu$-gradient occurs in the center at the very early stages of evolution (i.e. for $t \sim 10^{8} \mathrm{yr}$ ). At solar age, the diffusion is inhibited up to $M_{r} / M_{\odot} \sim 0.45$. The central properties of the mixed model are therefore very similar to the standard model properties, which nearly rules out turbulent mixing as a solution to the solar neutrino problem. The sound velocity of the two models are very close. The relative difference is less than $3.10^{-8}$ in the major part of the Sun.

The following results concerning the surface abundances of ${ }^{7} \mathrm{Li}$ and ${ }^{8} \mathrm{He}$ have been obtained: ${ }^{7} \mathrm{Li}$ is transported by turbulent diffusion from the bottom of the onter convective zone (OCZ) to the level where it is destroyed by nuclear reactions (i.e. at $T \sim 2.410^{6} \mathrm{~K}$ ): its surface abundance decreases with time. ${ }^{8} \mathrm{He}$ is transported from its peak of concentration (i.e. at $\left.M_{r} / M_{\odot} \sim 0.6\right)$ to the surface: its surface abundance increases with time. The moderate amount of mixing $\left(R e^{*} \sim 8 r / H_{p}\right.$ ) is able to satisfactorily explain the observed solar enhancement in ${ }^{8} \mathrm{He}$ (about $20 \%$ during the last $310^{\circ} \mathrm{yr}$ ). For the problem of ${ }^{7} \mathrm{Li}$, it appears that an amount of overshooting below the $\mathrm{OCZ}$ would be required to explain the observed solar ${ }^{7} \mathrm{Li}$ depletion (by a factor 100 during solar lifetime).

\section{OSCILLATIONS PROPERTIES}

The frequencies of $p$ modes of degree from 0 to 60 have been computed for the two models. These models are not precisely calibrated and the outer layers have not been calculated with high precision, so that the frequencies have been renormalized by a factor $\left(R_{\text {model }} / R_{\odot}\right)^{8 / 2}$ and the results must be considered as preliminary, in particular when the degree increases.

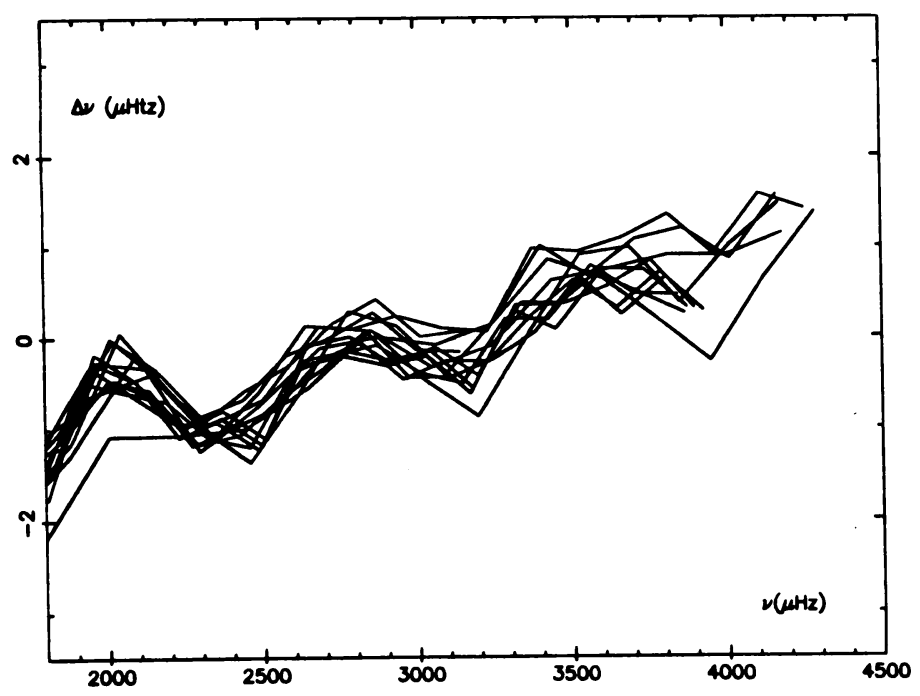

Figure 2: Differences of the $p$ modes frequencies of the two models scaled by the energy ratio $E_{l}(\nu) / E_{0}(\nu)$ are plotted as a fonction of the frequency, for $l=0$ to 60 . They are very small (from -1.5 to $1.5 \mu \mathrm{Hz}$ ) as can be expected from the small cound velocity difference between the two models. 


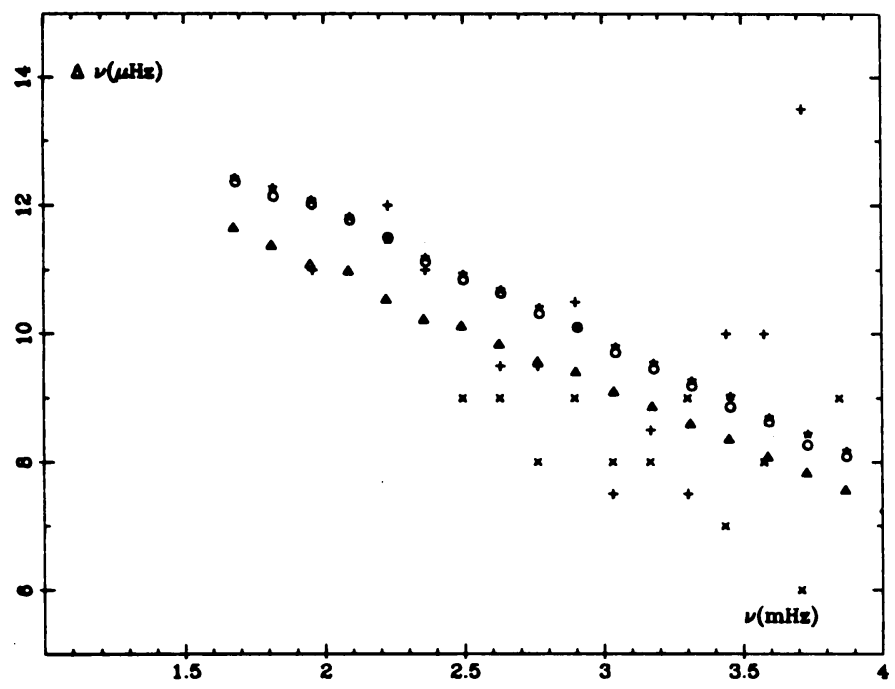

Figure 3: The splittings $\left(\nu_{l=2, n-1}-\nu_{l=0, n}\right)$ of low degree $p$ modes are plotted for the diffused $(\star)$ and standard $(0)$ models, for the standard Schatzman and Maeder's model $(\Delta)$ and for the observations ( $x$ : Claverie et al 1981; + :Grec et al 1980).

From Figures 2 and 3, it appears that the oscillations frequencies are not much modified by the introduction of the mixing by turbulent diffusion when inhibition by molecular weight gradient in the solar core is included. As it is expected, the frequency splitting between $l=0$ and $l=2$ modes is compatible with the observations.

\section{REFERENCES}

Berthomieu, G., Provost, J., Schatzman, E.: 1983, Proceedings of the Japan-France Colloquinm, Paris, octobre 1983, eds Y. Uchida et J.-C. Pecker, p. 189.

Claverie, A., Isaak, G.R., Mc Leod, C.P., Van der Raay, H.B. and Roca Cortes, T.: 1981, Nature 293,443.

Däppen, W.: 1981, Astron. Astrophys. 91, 212.

Fowler, W., Canghlan, G.R., Zimmerman, B.A.: 1975, Ann.Rev.Astron.Astrophys.13, 69 Grec, G., Fossat, E. and Pomerantz, M.: 1980, Nature, 288, 541.

Harris, M.J., Fowler, W.A., Canghlan, G.R., Zimmerman, B.A.: 1983, Ann. Rev. Astron. Astrophys. 13, 69.

Hopfinger, E.J., Browand, F.K., Gagne, Y.: 1982, J. Fluid. Mech.125,505.

Huebner, W.F., Merts, A.L., Magee, N.H.Jr., Argo, M.F.: 1977, Astrophysical Opacity Library, UC-34b.

Lebreton, Y., Maeder, A.: 1986a, Astron. Astrophys. 161,119.

Lebreton, Y., Maeder, A.: 1986b, Astron. Astrophys., in press.

Schatzman, E.: 1977, Astron. Astrophys. 56,211.

Schatzman, E., Maeder, A.: 1981, Astron. Astrophys. 96,1.

Zahn, J.P.: $1983,13^{\text {th }}$ Advanced Course of Saas-Fee, Observ. of Geneva. 